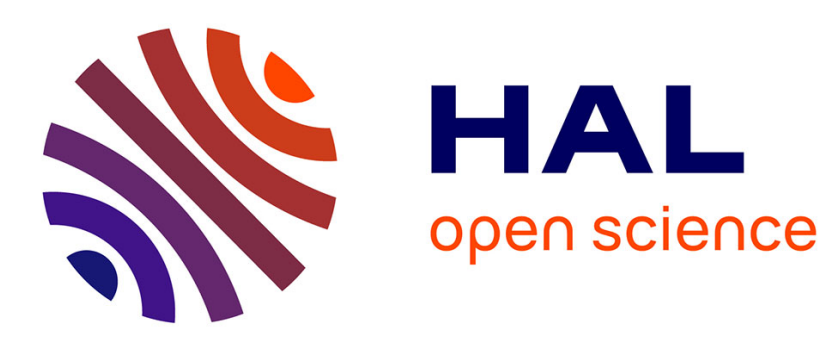

\title{
Courants de Foucault dans les pièces polaires d'une machine synchrone
}

\author{
F. Bouillault, A. Razek
}

\section{To cite this version:}

F. Bouillault, A. Razek. Courants de Foucault dans les pièces polaires d'une machine synchrone. Revue de Physique Appliquée, 1986, 21 (2), pp.181-185. 10.1051/rphysap:01986002102018100 . jpa00245423

\section{HAL Id: jpa-00245423 https://hal.science/jpa-00245423}

Submitted on 1 Jan 1986

HAL is a multi-disciplinary open access archive for the deposit and dissemination of scientific research documents, whether they are published or not. The documents may come from teaching and research institutions in France or abroad, or from public or private research centers.
L'archive ouverte pluridisciplinaire HAL, est destinée au dépôt et à la diffusion de documents scientifiques de niveau recherche, publiés ou non, émanant des établissements d'enseignement et de recherche français ou étrangers, des laboratoires publics ou privés. 


\title{
Courants de Foucault dans les pièces polaires d'une machine synchrone
}

\author{
F. Bouillault et A. Razek \\ Laboratoire de Génie Electrique de Paris, E.S.E., Univ. Paris VI et Paris XI, \\ L.A. no 127 au C.N.R.S., Plateau du Moulon, 91190 Gif sur Yvette, France
}

(Reçu le 8 juillet 1985, révisé le 15 octobre 1985, accepté le 17 octobre 1985)

\begin{abstract}
Résumé. - Cet article est consacré aux pertes supplémentaires créées par les courants de Foucault, se développant dans les parties massives d'un pôle saillant d'une machine synchrone alimentée par des ondes de courant chargées en harmoniques. Les résultats sont obtenus à partir de la résolution de l'équation de diffusion magnétique. Pour cela, la technique utilisée est la méthode des éléments finis, appliquée à un modèle bidimensionnel. Les effets de la fréquence des phénomènes sont étudiés ainsi que les distributions temporelle et spatiale des courants de Foucault dans le rotor. La réduction des pertes par un cuivrage du pôle est abordée. Les résultats sont relatifs à une machine de $10 \mathrm{~kW}$.
\end{abstract}

\begin{abstract}
This paper concerns an investigation of eddy currents and additional losses in solid parts of current fed salient pole synchronous machines. The results are obtained by the solution of the magnetic field diffusion equation. Eddy currents are determined using the finite elements method in a two dimensional machine section. The effects of working frequencies and the distributions of eddy-currents in space and in time are studied and discussed. The introduction of a distributed copper damper winding on the pole surface in the model is also discussed.
\end{abstract}

\section{Nomenclature}

$A$

potentiel vecteur

$v \quad$ reluctivité magnétique

$V \quad$ vitesse

$J_{0} \quad$ densité des courants de source

[A] vecteur composé des valeurs nodales de $A$

[I] vecteur composé des valeurs nodales du courant

$[A]^{n} \quad$ valeur de $[A]$ à l'instant $t=n \Delta t$

$\Delta t \quad$ pas de temps

$[S]^{n} \quad$ matrice de raideur à l'instant $t=n \Delta t$

$\theta=\omega t \quad \omega$ est la pulsation du fondamental des courants statoriques

\section{Introduction.}

Dans de nombreuses applications, les machines synchrones sont utilisées associées à des convertisseurs statiques [1, 2]. Dans ce cas, les courants statoriques ne sont pas sinusoïdaux; il y a alors, dans l'entrefer, des champs tournants asynchrones qui créent dans les parties conductrices du rotor des courants induits. $\mathrm{Ce}$ sera particulièrement le cas, si les rotors des machines comportent des parties massives [3]. L'utilisation de tels rotors est intéressante si l'on désire obtenir de faible coût de fabrication.
Les courants induits peuvent modifier considérablement les performances de la machine en créant des pertes supplémentaires dans le cuivre et dans le fer massif. Ainsi les pertes totales, créées dans une machine, dépassent leurs valeurs nominales lorsque la machine est alimentée par des courants statoriques non sinusoïdaux.

Ces pertes créent des échauffements et détériorent le rendement de la machine. Pour les faibles vitesses de rotation, les pertes sont plus faibles que pour des vitesses plus élevées, mais les échauffements sont très néfastes car la machine est très peu ventilée. Aussi faut-il tenir compte de ces pertes supplémentaires dans la conception de la machine. Une autre solution, économiquement moins intéressante, serait de déclasser la machine.

Le calcul des courants induits peut être obtenu à partir des équations électriques de la machine si les impédances des différents circuits sont connues. On tient compte alors de l'existence des courants de Foucault, en introduisant dans le schéma équivalent de la machine une impédance surfacique [4]. L'inconvénient majeur d'une telle démarche est qu'elle nous permet seulement d'avoir l'influence globale des courants de Foucault.

La connaissance de la répartition locale des cou- 
rants de Foucault nécessite la résolution de l'équation de diffusion magnétique [5]. Une telle équation aux dérivées partielles est un opérateur elliptique vis-à-vis des variations spatiales du potentiel vecteur et un opérateur parabolique vis-à-vis des variations temporelles. Dans une formulation plus large, il nous faut tenir compte du mouvement du rotor. Nous allons donc rappeler une formulation permettant de calculer la distribution du potentiel vecteur. Nous l'appliquerons à la modélisation d'une machine synchrone alimentée par des ondes de courant chargées en harmoniques.

\section{Formulation mathématique.}

Le potentiel vecteur magnétique dans un système électromagnétique avec des courants induits est obtenu à partir de l'équation de diffusion :

$$
\boldsymbol{\nabla} \wedge(v \boldsymbol{\nabla} \wedge \mathbf{A})=\mathbf{J}_{0}+\mathbf{J}_{\mathbf{F}}
$$

avec

$$
\mathbf{J}_{\mathbf{F}}=\sigma\left(-\frac{\partial \mathbf{A}}{\partial t}+\nabla \wedge(\mathbf{V} \wedge \mathbf{A})\right) .
$$

Dans le cas d'une résolution par éléments finis et d'un problème bidimensionnel, nous obtenons l'équation matricielle suivante :

$$
[S][A]_{t}=[I]_{t}-\left.[T] \frac{\mathrm{d}[A]}{\mathrm{d} t}\right|_{t} .
$$

La prise en compte du mouvement dans le modèle est facilité si l'on choisit convenablement les repères de calcul [6]. Dans de telles conditions, l'opérateur "dérivation par rapport au temps " dans (3) peut être approximé par l'algorithme suivant :

$\beta\left[\frac{\mathrm{d} A}{\mathrm{~d} t}\right]_{t+\Delta t}+(1-\beta)\left[\frac{\mathrm{d} A}{\mathrm{~d} t}\right]_{t}=\frac{[A]_{t+\Delta t}-[A]_{t}}{\Delta t}$

avec $0<\beta \leqq 1$.

La meilleure précision est obtenue pour $\beta=0,5$, mais dans ce cas, la convergence est très lente. Aussi peut-on commencer les calculs avec $\beta=1$ et affiner par la suite les résultats avec $\beta=0,5$.

D'autre part, le changement de géométrie pendant la rotation peut être considéré en utilisant dans l'entrefer un macro-élément [6-8]. Dans ce cas, la matrice [S] dans l'équation (3) peut être considérée comme l'assemblage de 3 matrices élémentaires, la première représentant le stator, la deuxième l'entrefer et la troisième le rotor.

L'équation (3) sous sa forme discrétisée devient

$$
\begin{aligned}
\left(\beta[S]^{n+1}+\frac{[T]}{\Delta t}\right)[A]^{n+1} & =\beta[I]^{n+1}+(1-\beta)[I]^{n}- \\
& -\left((1-\beta)[S]^{n}+\frac{[T]}{\Delta t}\right)[A]^{n} .
\end{aligned}
$$

\section{Calculs numériques.}

Le modèle décrit ci-dessus est particulièrement bien adapté pour étudier les phénomènes existant aux basses fréquences. Nous avons déterminé les courants de Foucault et les pertes créées dans les parties massives (calotte polaire) d'une machine synchrone dont les caractéristiques sont les suivantes : $10 \mathrm{~kW}, 320 \mathrm{~V}$, 4 pôles, $3000 \mathrm{tr} / \mathrm{min}$. Les courants de Foucault sont déterminés dans le cas d'une alimentation en courant de la machine [2].

\section{1 HYPOTHÈSES SIMPLIFICATRICES.}

- Les courants sont supposés dirigés suivant l'axe de la machine, ainsi l'étude peut être faite avec un modèle bidimensionnel [9].

- Le circuit magnétique du stator étant feuilleté, la conductivité électrique est prise égale à zéro.

- L'état de saturation magnétique est globalement considéré en prenant une perméabilité constante dans le rotor et dans le stator. L'état de saturation locale pourrait être pris en compte mais le temps et le coût du calcul deviennent prohibitifs [5].

- Les courants statoriques considérés sont représentés sur la figure 1 .

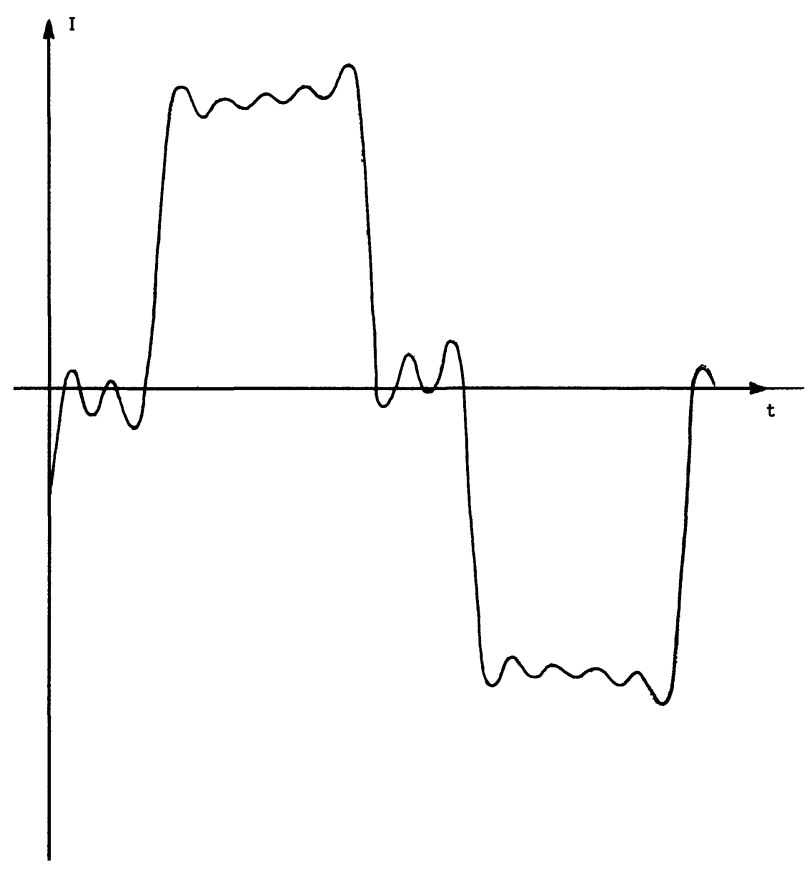

Fig. 1. - Courants statoriques considérés dans la modélisation.

[Considered armature phase current wave form.]

L'amplitude de ces courants est choisie de telle façon que la valeur de leur fondamental soit égale à la valeur nominale du courant statorique de la machine. De plus les harmoniques considérées sont d'ordre 5 , $7,11,13$; les harmoniques d'ordre supérieur existant 
dans les courants délivrés par un convertisseur sont négligés. Comme les courants de Foucault induits dans le rotor sont dus aux harmoniques de courants statoriques, les courants de source utilisés dans notre modèle sont constitués uniquement des harmoniques. Dans ce cas, la perméabilité statorique est prise égale à une perméabilité moyenne entre la perméabilité obtenue dans la partie linéaire de la courbe $B(H)$ et la perméabilité à la saturation. Dans le rotor, on considère une perméabilité différentielle moyenne.

Le domaine de résolution comporte 1053 nœuds et 1976 triangles du premier ordre. La partie uniforme de l'entrefer est représentée par un macro-élément de 76 nouds. Le pas de calcul $\Delta t$ est égal à $1 /(180 f)$ où $f$ est la fréquence des courants statoriques. La convergence numérique est alors pratiquement atteinte pour un temps égal à $30 \Delta t$.

3.2 Résultats. - Le calcul des pertes et des courants de Foucault a été fait pour 2 fréquences 2 et $16 \mathrm{~Hz}$. La figure 2 donne le tracé de lignes de champ pour la fréquence de $16 \mathrm{~Hz}$. Pour cette fréquence, le maillage est tel que l'épaisseur de peau relative à l'harmonique de rang le plus élevé $(13 \times 16 \mathrm{~Hz})$ correspond à la hauteur d'une maille. Pour des fréquences plus élevées, le nombre de nœuds nécessaire pour avoir une précision suffisante ainsi que le coût du calcul deviennent trop importants. Le tracé des lignes de champs montre que les courants de Foucault ont pour effet de refouler le flux magnétique à la surface des matériaux (ce tracé fait apparaître quelques imprécisions sur la détermination des équipotentielles vecteurs; certaines sont dues à la discrétisation en éléments triangulaires, d'autres à la technique de tracé des équipotentielles).

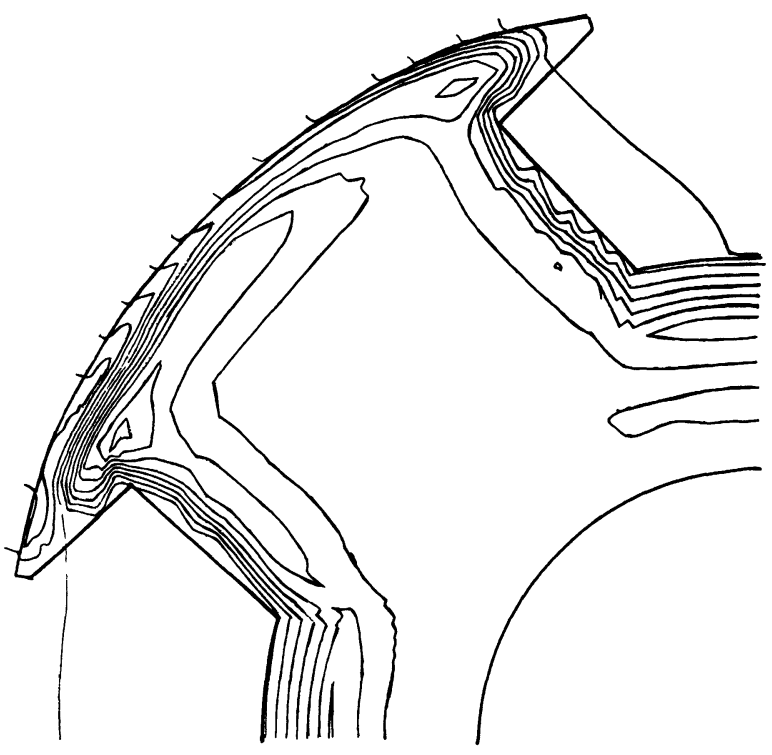

Fig. 2. - Tracé des lignes d'induction - fréquence $16 \mathrm{~Hz}$. [Flux distribution - frequency $16 \mathrm{~Hz}$.]

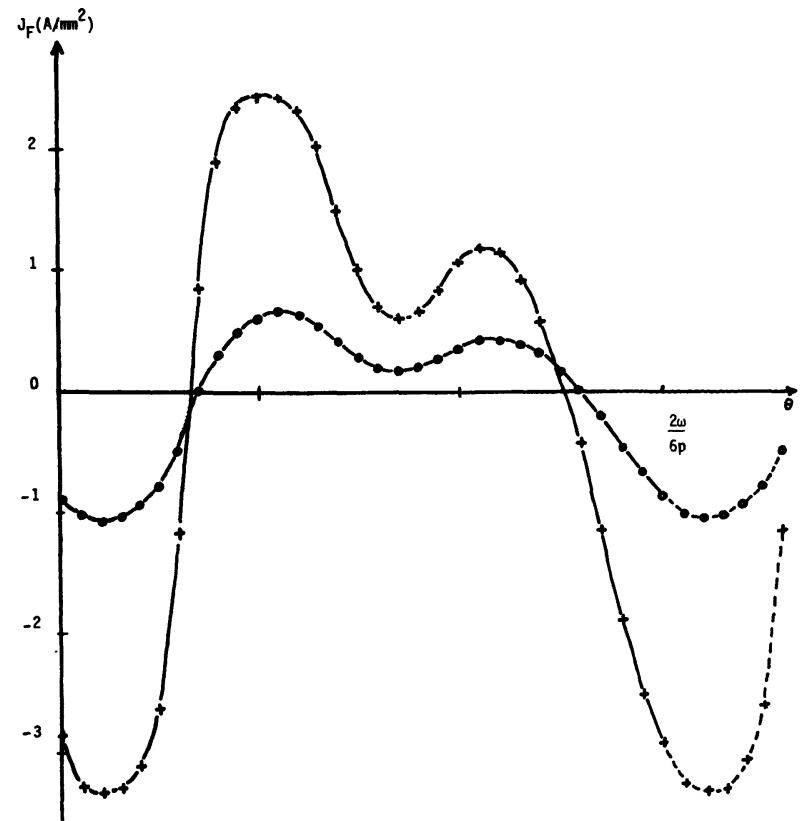

Fig. 3. - Densité des courants de Foucault en fonction de l'angle de rotation du rotor $\theta=\omega t / p$ en un point à la surface du pôle $+-+-16 \mathrm{~Hz} ; \mathrm{O}-\mathrm{O}-2 \mathrm{~Hz}$.

[Eddy current density on the pole surface (centre) as a function of angle $\theta=\omega t / p$.]

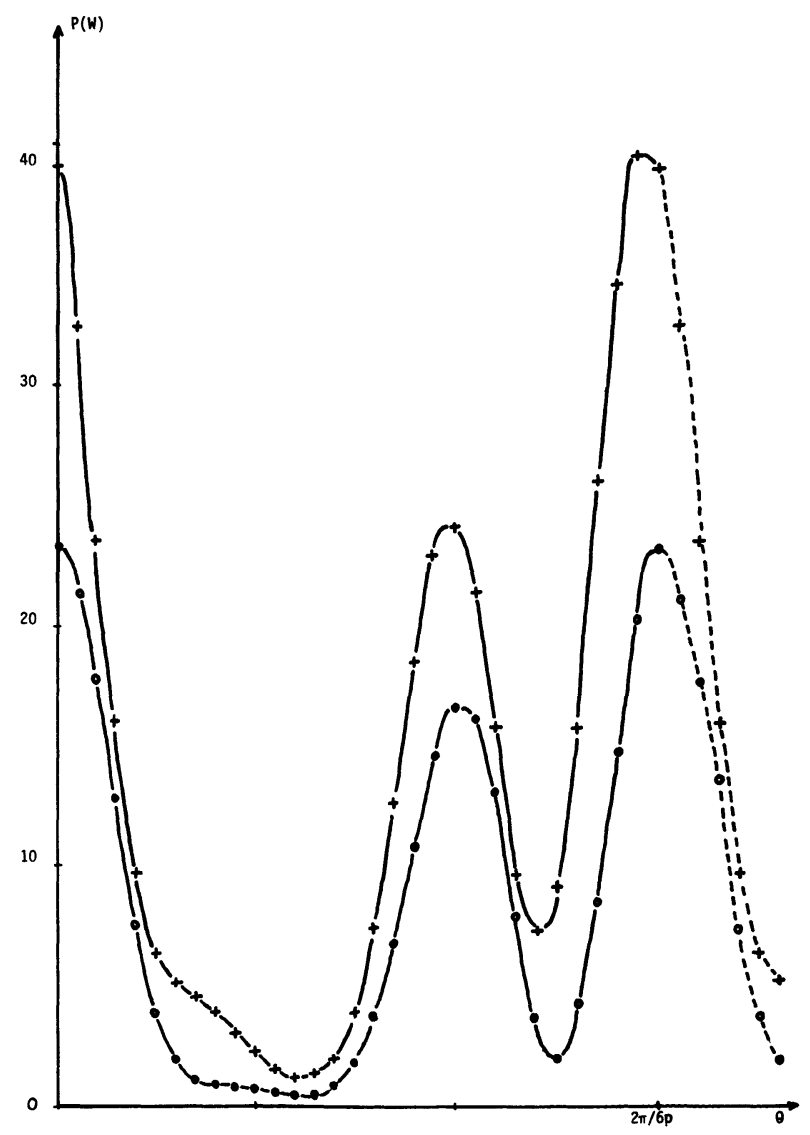

Fig. 4. - Pertes totales dans le pôle en fonction de l'angle $\theta$ pour des fréquences de fonctionnement $+-+-16 \mathrm{~Hz}$; $\mathrm{O}-\mathrm{O}-2 \mathrm{~Hz}$

[Total losses function of angle $\theta$.] 
La figure 3 représente la densité des courants induits en un point situé au centre de la surface du pôle. La figure 4 donne les pertes totales en fonction de l'angle $\theta=\omega t$. La valeur moyenne de ces pertes à $16 \mathrm{~Hz}$ est de $16 \mathrm{~W}$, soit $1 \%$ de la puissance de la machine à cette fréquence (il est à noter que le rendement d'une machine synchrone alimentée par des courants statoriques sinusoïdaux est d'environ $98 \%$ ). L'analyse spectrale des courbes de la figure 4 donne, outre la composante continue, les harmoniques 6,12 . 18 , et 24 . Si le rotor était lisse, les harmoniques de courants statoriques 5 , $7,11,13$ produiraient deux champs tournants l'un à la pulsation $6 \omega$, l'autre à la pulsation $12 \omega$ vis-à-vis du rotor [4]. L'analyse spectrale des pertes dues à ces deux champs donnerait une composante continue et une composante alternative à la pulsation (12-6) $\omega$. Dans le cas présent, la saillance augmente la composante continue des pertes et introduit des harmoniques 12,18 et 24 . Il est évident que l'amplitude des harmoniques et naturellement la forme des courbes des figures 3 et 4 dépendent de la structure du pôle.

Par ailleurs, des essais thermiques ont été réalisés sur cette machine [10]. Les pertes moyennes pour la fréquence de $16 \mathrm{~Hz}$, ont été évaluées à $28 \mathrm{~W}$. La différence avec les pertes calculées peut s'expliquer par l'imprécision des résultats pour de faible puissance dissipée et par la difficulté de séparer, à partir des essais, les pertes dues aux harmoniques de courant et celles dues aux harmoniques d'encoches. Les pertes,

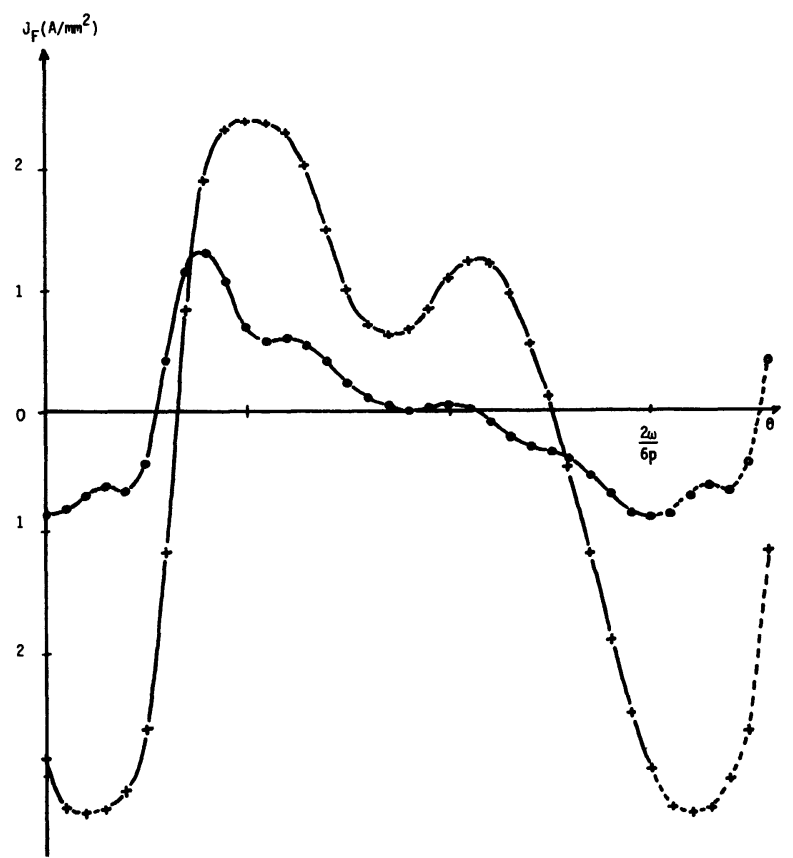

Fig. 5. - Densité de courant en un point à la surface du pôle en fonction de l'angle de rotation $\theta$ pour une fréquence de $16 \mathrm{~Hz},+-+-$ rotor classique; $0-0-$ rotor cuivré.

[Eddy current density on the centre of the pole surface $16 \mathrm{~Hz} .+-+-$ classical rotor; $\mathrm{O}-\mathrm{O}-$ with copper cladding.]

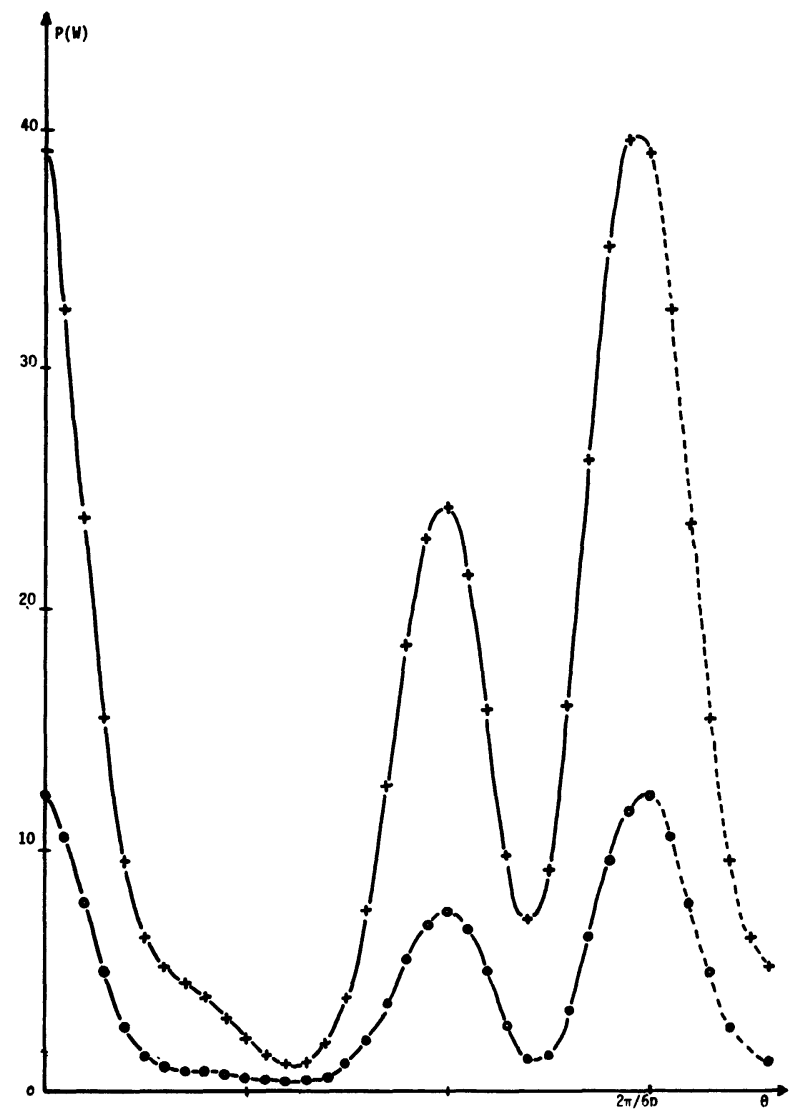

Fig. 6. - Pertes totales en fonction de l'angle de rotation $\theta$ pour une fréquence de $16 \mathrm{~Hz} .+-+-$ rotor non cuivré; O-O- rotor cuivré.

[Total losses function of angle $\theta .+-+-$ without copper claddıng; O-O- with copper cladding.]

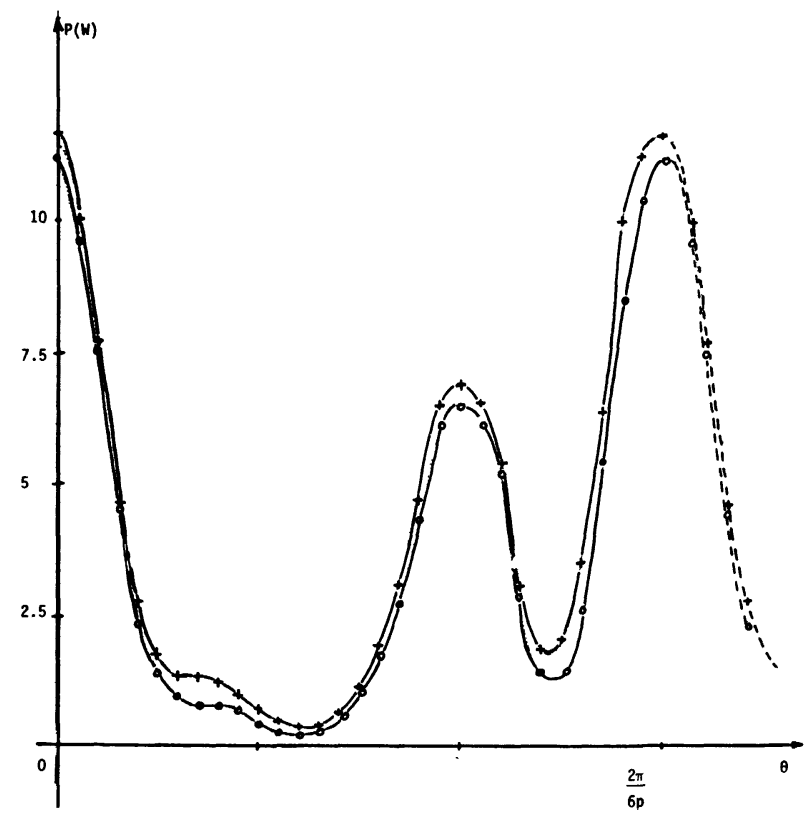

Fig. 7. - Pertes totales en fonction de l'angle de rotation $\theta$ pour une fréquence de $2 \mathrm{~Hz}$. O-O- rotor cuivré; +-+- rotor non cuivré.

[Total losses function of angle $\theta$ at $2 \mathrm{~Hz}$. O-O- with copper cladding; +-+- without copper cladding.] 
relatives aux harmoniques d'encoches, ont pour origine principale les variations du champ inducteur qui n'est pas pris en compte dans notre modélisation.

3.3 EFFET DU CUIVRAGE DU PÔLE. - Il a été montré que les pertes créées dans un pôle massif peuvent être réduites si l'on cuivre le pôle [4]. Pour étudier ce phénomène, nous avons regardé l'influence d'une couche de cuivre de $1 \mathrm{~mm}$ recouvrant le pôle. La figure 5 montre l'évolution de la densité des courants induits à la surface du pôle; le cuivrage du pôle permet dans ce cas de diminuer l'amplitude des courants induits. L'efficacité du cuivrage du pôle dépendra de la fréquence des phénomènes (Figs. 6 et 7). Une étude plus complète sur ce phénomène [11] montre qu'il faut également adapter l'épaisseur de la couche de cuivre à la fréquence des courants induits.

\section{Conclusion.}

Dans cet article, les courants de Foucault et les pertes supplémentaires créées dans les parties massives d'une machine synchrone sont localement calculées. Les effets de la fréquence et de la saillance du pôle sont analysés. La méthode numérique utilisée permet de considérer le mouvement et la géométrie réelle. Les résultats numériques semblent en accord avec les résultats expérimentaux. Le modèle utilisé pour notre étude peut être intéressant pour améliorer les connaissances sur les phénomènes dynamiques existant aux basses fréquences.

\section{Bibliographie}

[1] Franklin, P. W., Theory of the three phase salient pole type generator with bridge rectifier, Trans. I.E.E.E. PAS-91 (1972) 1960-1975.

[2] Chassande, J. P., Razek, A., Poloujadoff, M. and LAUMAND, A., Various practical results concerning the operation of inverter fed self controlled synchronous machines, Trans. I.E.E.E. PAS-101, (1982) 4649-4655.

[3] Lechat, D., Rialland, J. F. et Bonnefille, R., Analyse des pertes rotoriques dissipées dans les machines synchrones associées à des convertisseurs statiques, Revue Phys. Appl. 19 (1984) 395-402.

[4] RAzeK, A., Additional losses in solid-rotor salient pole synchronous machines associated with static convertors, E.M.E. 5 (1980) 101-111.

[5] Bouillault, F. and RazeK, A., Dynamic model for eddy current calculation in saturated electric machines, Trans. I.E.E.E. MAG-19 (1983) 26282631.

[6] Bouillault, F. et RazeK, A., Prise en compte du mouvement dans la détermination numérique des courants de Foucault dans une structure électromagnétique, Revue Phys. Appl. 18 (1983) 103-106.
[7] RazeK, A., Coulomb, J. C., Feliachi, M. and SabonNADIÈRE, J. C., Conception of an air-gap element for dynamic analysis of the electromagnetic field in electric machines, Trans. I.E.E.E. MAG-18 (1982) 655-659.

[8] Bouillault, F. and RazeK, A., Numerical calculation of eddy currents in mobil electromagnetic systems, Proc. U.P.E.C., Guildford, 1983.

[9] Chari, M. V. K. and Silvester, P. P., Finite element in electrical and magnetic field problems (John Wiley \& Sons, New York) 1980.

[10] Milimonfarad, D., Contribution à la modélisation thermique des machines synchrones à rotor massif et $\grave{a}$ pôles saillants - Applications aux moteurs autopilotés. Thèse de Docteur-Ingénieur, Université Pierre et Marie Curie - 1984.

[11] Darnand, H. and Grellet, G., Pole face losses of machines with solid copper-faced shoes and fed by non sinuso-dal current waves, Proc. of I.C.E.M., pp. 853-856 Budapest 82 . 